\title{
Additional list of species of aquatic macrophytes in the lower basin of the Xingu River
}

\author{
Abe, DS. ${ }^{*}$, Sidagis-Galli, C. ${ }^{a}$, Matsumura-Tundisi, T. ${ }^{a}$, Tundisi, JEM. ${ }^{a}$, \\ Blanco, FP. ${ }^{a}$, Faria, CRL. ${ }^{a}$ and Tundisi, JG. ${ }^{a}$ \\ anstituto Internacional de Ecologia e Gerenciamento Ambiental - IIEGA, Rua Bento Carlos, 750, \\ Centro, CEP 13560-660, São Carlos, SP, Brazil \\ *e-mail: donatoabe@iie.com.br
}

Received: July 10, 2015 - Accepted: August 10, 2015 - Distributed: August 31, 2015

(With 2 figures)

\begin{abstract}
In this paper the authors present an additional list of aquatic macrophytes in the lower basin of the Xingu River.

Keywords: macrophytes, lower Xingu River.
\end{abstract}

\section{Lista adicional de macrófitas aquáticas do baixo Rio Xingu}

\begin{abstract}
Resumo
Neste trabalho os autores apresentam uma lista adicional de macrófitas aquáticas no baixo Rio Xingu.
\end{abstract}

Palavras-chave: macrófitas, baixo Rio Xingu.

\section{Introduction}

The lower stretch of the Xingu River is characterized by a diversity of habitats, with large uneven rapids, a series of anastomosing channels, oxbow lakes and high amount of tributaries. This heterogeneity generates hydrological and limnological conditions that allow the occurrence of a variety of aquatic macrophytes.

In the article by Medeiros et al. (2015) presented in this special issue, the authors also conducted a survey of the species of aquatic weeds that occur in the same region. However, in this study the authors gave greater emphasis on closely related species to water bodies, since the main objective was to evaluate the species with great potential for infestation and that could cause problems in areas of the reservoirs under construction in the Xingu River.

In the present study, the sampling efforts were directed to a wider area including wetlands, parafluvial zone of the Xingu River, as well as the edges of islands and stretches of the Xingu river rapids. Therefore, it is a complement of the previous survey conducted by Medeiros et al. (2015).

\section{Methods}

This survey is part of the monitoring program that is being conducted in the influence areas of the Belo Monte hydroelectric dam. Sampling of aquatic macrophytes was carried out in four campaigns, considering one full hydrological cycle: October of 2014 (dry), January (flood), April (full) and July of 2015 (ebb) (Figure 1).

The species list followed the classification of families proposed by APG III (2009) for angiosperms, by Smith et al. (2006) for pteridophytes, and Buck and Goffinet (2000) for bryophytes. Plant names and respective authors were checked at the data bank of the Botanical Garden o Rio de Janeiro (Reflora, 2015).

A total of 189 species of aquatic macrophytes were observed (Table 1). The most represented families were Fabaceae (27 species), Cyperaceae (26 species), Poaceae (22 species) and Rubiaceae (10 species) (Figure 2). Amphibious forms were dominant $(60 \%)$, followed by emergent plant species (26\%), while free-floating were represented by $3 \%$ and rooted-floating by $3 \%$. Considering the plant families, Moura Júnior et al. (2015) also found Poaceae, Cyperaceae and Fabaceae as the most representative families in a checklist of aquatic macrophytes carried out for the Northern region of Brazil. These authors also observed an unprecedent richness of Podostemaceae due to their efforts on favorable habitats, a procedure not performed in the present study. Nevertheless, five species of Podostemaceae were observed in stretches of rapids in the Xingu River in the present study, most belonging to the genus Mourera, and the species Mourera fluviatilis appears in the list of endangered species of flora (Brasil, 2008) (Figure 2). 


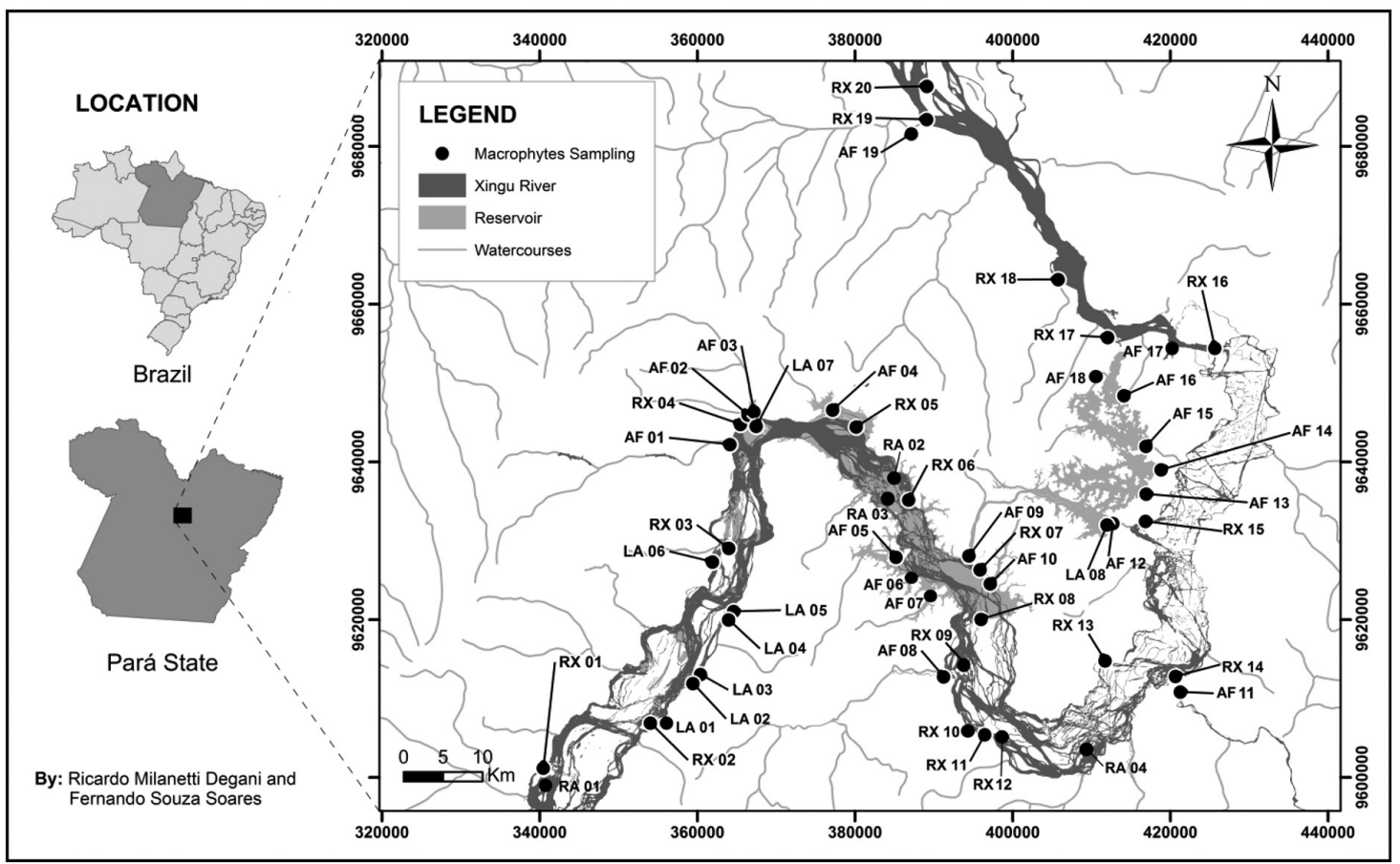

Figure 1. Map of the lower basin of the Xingu River showing the sampling stations of aquatic macrophytes in the period between December 2011 and April 2015.

Table 1. List of families, species, life forms and habitats of aquatic macrophytes in riverine landscapes of the middle Xingu River recorded from October 2014 to July 2015.

\begin{tabular}{|c|c|c|}
\hline Family/Species & Life form & Habitat \\
\hline \multicolumn{3}{|l|}{ Algae } \\
\hline \multicolumn{3}{|l|}{ Charophyceae } \\
\hline Chara sp. & Rooted submerged & $\mathrm{AF}$ \\
\hline \multicolumn{3}{|l|}{ Briophyte } \\
\hline \multicolumn{3}{|l|}{ Ricciaceae } \\
\hline Ricciocarpos natans (L.) Corda & Free floating & LA \\
\hline \multicolumn{3}{|l|}{ Pteridophyte } \\
\hline \multicolumn{3}{|l|}{ Dennstaedtiaceae } \\
\hline Pteridium arachnoideum (Kaulf.) Maxon & Amphibious & XR \\
\hline \multicolumn{3}{|l|}{ Thelypteridaceae } \\
\hline Thelipteris serrata (Cav.) Alston & Emergent & $\mathrm{LA}, \mathrm{AF}$ \\
\hline \multicolumn{3}{|l|}{ Salviniaceae } \\
\hline Azolla filiculoides Lam. & Free floating & LA \\
\hline Salvinia auriculata Aubl. & Free floating & $\mathrm{LA}, \mathrm{AF}$ \\
\hline \multicolumn{3}{|l|}{ Angiosperm } \\
\hline \multicolumn{3}{|l|}{ Alismataceae } \\
\hline Echinodorus macrophyllus (Kunth) Micheli subsp. Scaber & Emergent & LA \\
\hline \multicolumn{3}{|l|}{ Amaranthaceae } \\
\hline Alternanthera brasiliana (L.) Kuntze & Amphibious & $\mathrm{LA}, \mathrm{XR}, \mathrm{AF}$ \\
\hline Alternanthera tenella Colla & Amphibious & $\mathrm{AF}$ \\
\hline Amaranthus viridis $\mathrm{L}$. & Amphibious & $\mathrm{XR}$ \\
\hline Chamissoa altissima (Jacq.) Kunth & Amphibious & $\mathrm{XR}$ \\
\hline Gomphrena celosioides Mart. & Amphibious & TR \\
\hline \multicolumn{3}{|l|}{ Anacardiaceae } \\
\hline Tapirira guianensis Aubl. & Amphibious & TR \\
\hline
\end{tabular}

LA: lagoons; XR: Xingu River; AF: affluents. 
Table 1. Continued...

\begin{tabular}{|c|c|c|}
\hline Family/Species & Life form & Habitat \\
\hline \multicolumn{3}{|l|}{ Annonaceae } \\
\hline Oxandra riedeliana $\mathrm{R}$. E. Fr. & Amphibious & LA \\
\hline \multicolumn{3}{|l|}{ Apocynaceae } \\
\hline Cynanchum montevidense Spreng. & Amphibious & LA, XR \\
\hline Rhabdadenia biflora (Jacq.) Müll. Arg. & Amphibious & $\mathrm{XR}$ \\
\hline \multicolumn{3}{|l|}{ Araceae } \\
\hline Montrichardia linifera (Arruda) Schott & Emergent & LA, XR \\
\hline Pistia stratiotes $\mathrm{L}$. & Free floating & LA, XR \\
\hline \multicolumn{3}{|l|}{ Arecaceae } \\
\hline Bactris maraja Mart. & Amphibious & LA, XR, TR \\
\hline \multicolumn{3}{|l|}{ Asteraceae } \\
\hline Acanthospermum australe (Loefl.) Kuntze & Amphibious & $\mathrm{XR}, \mathrm{AF}$ \\
\hline Eclypta prostrata (L.) L. & Emergent & $\mathrm{LA}, \mathrm{XR}, \mathrm{AF}$ \\
\hline Emilia sonchifolia (L.) DC. ex Wight & Amphibious & $\mathrm{AF}$ \\
\hline Mikania cordifolia (L. f.) Wild. & Emergent & $\mathrm{LA}, \mathrm{XR}, \mathrm{AF}$ \\
\hline Sphagneticola trilobata (L.) Pruski & Amphibious & $\mathrm{AF}$ \\
\hline Trichospira verticillata (L.) S. F. Blake & Amphibious & LA, XR \\
\hline Wedelia calycina Rich. & Amphibious & LA, XR \\
\hline \multicolumn{3}{|l|}{ Bignoniaceae } \\
\hline Bignonia sp. & Amphibious & $\mathrm{XR}$ \\
\hline Tanaecium pyramidatum (Rich.) L. G. Lohmann & Amphibious & $\mathrm{XR}, \mathrm{TR}$ \\
\hline \multicolumn{3}{|l|}{ Boraginaceae } \\
\hline Euploca procumbens (Mill.) Diane \& Hilger & Emergent & LA, XR \\
\hline Heliotropium indicum (L.) & Emergent & LA, XR \\
\hline \multicolumn{3}{|l|}{ Cabombaceae } \\
\hline Cabomba aquatica Aubl. & Rooted submerged & $\mathrm{AF}$ \\
\hline \multicolumn{3}{|l|}{ Commelinaceae } \\
\hline Commelina bengalensis $\mathrm{L}$. & Amphibious & LA, XR \\
\hline \multicolumn{3}{|l|}{ Cannabaceae } \\
\hline Trema micrantha $(\mathrm{L}$.$) Blume$ & Amphibious & $\mathrm{AF}$ \\
\hline \multicolumn{3}{|l|}{ Convolvulaceae } \\
\hline Iseia luxurians (Moric.) O’Donnell & Amphibious & LA, XR \\
\hline Ipomoea setifera Poir & Emergent or Amphibious & XR \\
\hline \multicolumn{3}{|l|}{ Cucurbitaceae } \\
\hline Luffa operculata (L.) Cogn. & Amphibious & $\mathrm{XR}, \mathrm{AF}$ \\
\hline \multicolumn{3}{|l|}{ Cyperaceae } \\
\hline Cyperus aggregatus (Wild.) Endl. & Amphibious & LA \\
\hline Cyperus articulatus L. & Amphibious & $\mathrm{XR}$ \\
\hline Cyperus digitatus Roxb. & Amphibious & $\mathrm{LA}, \mathrm{AF}$ \\
\hline Cyperus distans $\mathrm{L}$. & Amphibious & $\mathrm{LA}, \mathrm{AF}$ \\
\hline Cyperus esculentus $\mathrm{L}$. & Amphibious & $\mathrm{AF}$ \\
\hline Cyperus exaltatus Retz. & Amphibious & LA \\
\hline Cyperus giganteus Vahl & Amphibious & LA, XR \\
\hline Cyperus iria $\mathrm{L}$. & Amphibious & $\mathrm{AF}$ \\
\hline Cyperus luzulae (L.) Rottb. ex Retz. & Amphibious & $\mathrm{LA}, \mathrm{XR}, \mathrm{AF}$ \\
\hline Cyperus odoratus L. & Amphibious & $\mathrm{LA}, \mathrm{XR}, \mathrm{AF}$ \\
\hline Cyperus sphacelatus Rottb. & Amphibious & $\mathrm{XR}, \mathrm{AF}$ \\
\hline Cyperus surinamensis Rottb. & Amphibious & $\mathrm{LA}, \mathrm{XR}, \mathrm{AF}$ \\
\hline Eleocharis interstincta (Vahl) Roem. \& Schult. & Amphibious & LA \\
\hline Eleocharis minima Kunth & Emergent/Amphibious & LA \\
\hline Fimbristylis dichotoma (L.) Vahl & Amphibious & $\mathrm{AF}$ \\
\hline
\end{tabular}

LA: lagoons; XR: Xingu River; AF: affluents. 
Table 1. Continued...

\begin{tabular}{|c|c|c|}
\hline Family/Species & Life form & Habitat \\
\hline Fimbristylis miliacea (L.) Vahl. & Amphibious & $\mathrm{LA}, \mathrm{AF}$ \\
\hline Fuirena umbellata Rottb. & Amphibious & $\mathrm{LA}, \mathrm{AF}$ \\
\hline Kyllinga vaginata Lam. & Amphibious & $\mathrm{AF}$ \\
\hline Oxycarium cubense (Poepp. \& Kunth) Lye & Emergent & $\mathrm{LA}, \mathrm{XR}, \mathrm{AF}$ \\
\hline Pycreus aff. polystachyos (Rottb.) P. Beauv. & Amphibious & XR \\
\hline Pycreus lanceolatus Poir. C.B. Clarke & Amphibious & $\mathrm{LA}, \mathrm{XR}, \mathrm{AF}$ \\
\hline Rhynchospora nervosa (Vahl) Boeckeler) T. Koyama & Emergent & $\mathrm{AF}$ \\
\hline Scleria gartneri Rad. & Amphibious & XR \\
\hline Scleria microcarpa Nees ex Kunth & Amphibious & LA \\
\hline Scleria mitis P.J. Bergius & Amphibious & $\mathrm{AF}$ \\
\hline Scleria secans (L.) Urb. & Amphibious & LA, XR, AF \\
\hline \multicolumn{3}{|l|}{ Euphorbiaceae } \\
\hline Caperonia castaneifolia (L.) A. St.-Hill. & Emergent & $\mathrm{LA}, \mathrm{XR}, \mathrm{AF}$ \\
\hline Croton trinitatis Millsp. & Amphibious & $\mathrm{LA}, \mathrm{AF}$ \\
\hline Euphorbia hyssopifolia L. & Emergent & XR \\
\hline Sapium glandulosum (L.) Morong & Emergent & $\mathrm{XR}, \mathrm{AF}$ \\
\hline \multicolumn{3}{|l|}{ Fabaceae } \\
\hline Acacia plumosa Martius ex Colla & Amphibious & $\mathrm{AF}$ \\
\hline Aeschynomene brasiliana DC. & Emergent & $\mathrm{AF}$ \\
\hline Calopogonium mucunoides Desv. & Amphibious & $\mathrm{AF}$ \\
\hline Campsiandra angustifolia Spruce ex Benth. & Emergent & $\mathrm{XR}, \mathrm{AF}$ \\
\hline Chamaecrista diphylla L. Greene & Amphibious & LA \\
\hline Chamaecrista nictitans (L.) Moench & Amphibious & $\mathrm{XR}, \mathrm{AF}$ \\
\hline Clitoria laurifolia Poir. & Amphibious/epiphyta & $\mathrm{LA}, \mathrm{XR}, \mathrm{AF}$ \\
\hline Calopogonium mucunoides Desv. & Amphibious & $\mathrm{AF}$ \\
\hline Crotalaria lanceolata E. Mey. & Amphibious & $\mathrm{AF}$ \\
\hline Crotalaria micans Link & Amphibious & $\mathrm{AF}$ \\
\hline Crotalaria palida Aiton & Amphibious & $\mathrm{AF}$ \\
\hline Crotalaria spectabilis Roth & Amphibious & $\mathrm{AF}$ \\
\hline Cymbosema roseum Benth. & Amphibious & $\mathrm{XR}$ \\
\hline Desmodium barbatum (L.) Benth. & Amphibious & $\mathrm{AF}$ \\
\hline Indigofera lespedezioides Kunth & Amphibious & LA, AF \\
\hline Macrolobium acaciifolium (Benth.) Benth. & Amphibious & XR \\
\hline Macroptilium gracile (Poepp). ex Willd. & Amphibious & $\mathrm{AF}$ \\
\hline Mimosa pigra L. & Amphibious & XR \\
\hline Mimosa pudica L. & Emergent & $\mathrm{LA}, \mathrm{XR}, \mathrm{AF}$ \\
\hline Senna obtusifolia (L.) H.S. Irwin \& Barneby & Emergent & $\mathrm{AF}$ \\
\hline Senna reticulata Wild.) H. S. Irwin \& Barneby & Amphibious & $\mathrm{AF}$ \\
\hline Sesbania exasperata Kunth & Amphibious & XR \\
\hline Stylosanthes viscosa (L.) Sw. & Amphibious & $\mathrm{AF}$ \\
\hline Teramnus volubilis $\mathrm{Sw}$. & Amphibious & $\mathrm{AF}$ \\
\hline Vigna lasiocarpa (Mart. Ex Benth.) Verdc. & Amphibious & LA \\
\hline Vigna longifolia (Benth.) Verdc. & Amphibious & LA \\
\hline Zornia latifolia Sm. & Amphibious & LA \\
\hline \multicolumn{3}{|l|}{ Gentianaceae } \\
\hline Coutoubea spicata Aubl. & Amphibious & $\mathrm{AF}$ \\
\hline \multicolumn{3}{|l|}{ Hydroleaceae } \\
\hline Hydrolea spinosa L. & Emergent & LA \\
\hline \multicolumn{3}{|l|}{ Hypericaceae } \\
\hline Hypericum brasiliense Choisy & Emergent & LA AF \\
\hline Vismia sp. & Amphibious & $\mathrm{XR}, \mathrm{AF}$ \\
\hline
\end{tabular}

LA: lagoons; XR: Xingu River; AF: affluents. 
Table 1. Continued...

\begin{tabular}{|c|c|c|}
\hline Family/Species & Life form & Habitat \\
\hline \multicolumn{3}{|l|}{ Lamiaceae } \\
\hline Cantinoa mutabilis (Rich.) Harley \& J.F.B. Pastore & Amphibious & $\mathrm{XI}, \mathrm{AF}$ \\
\hline Hyptis atrorubens Poit. & Emergent & LA, XR, AF \\
\hline Hyptis brevipes Poit. & Emergent & $\mathrm{AF}$ \\
\hline \multicolumn{3}{|l|}{ Lindernaceae } \\
\hline Lindernia crustacea (L.) F. Muell. & Amphibious & $\mathrm{LA}, \mathrm{XR}, \mathrm{AF}$ \\
\hline \multicolumn{3}{|l|}{ Lythraceae } \\
\hline Cuphea fruticosa Spreng. & Amphibious & $\mathrm{XR}$ \\
\hline \multicolumn{3}{|l|}{ Malvaceae } \\
\hline Byttneria genistella Tr. et. Pl. & Emergent & $\mathrm{XR}$ \\
\hline Hibiscus furcellatus Desr. & Amphibious & $\mathrm{LA}, \mathrm{XR}, \mathrm{AF}$ \\
\hline Malachra radiata $(\mathrm{L}.) \mathrm{L}$. & Emergent & $\mathrm{AF}$ \\
\hline Sida acuta Burm. f. & Amphibious & $\mathrm{AF}$ \\
\hline Sida rhombifolia $\mathrm{L}$. & Amphibious & LA \\
\hline Sida santaremensis $\mathrm{H}$. Monteiro & Emergent & $\mathrm{AF}$ \\
\hline Urena lobata $\mathrm{L}$. & Emergent & $\mathrm{LA}, \mathrm{AF}$ \\
\hline \multicolumn{3}{|l|}{ Marantaceae } \\
\hline Thalia geniculata L. & Emergent & LA \\
\hline \multicolumn{3}{|l|}{ Melastomataceae } \\
\hline Miconia sp. & Emergent & $\mathrm{AF}$ \\
\hline Rhynchanthera dichotoma (Desr.) DC. & Amphibious & $\mathrm{AF}$ \\
\hline \multicolumn{3}{|l|}{ Molluginaceae } \\
\hline Glinus radiatus (Ruiz \& Pav.) Rohrb. & Emergent & $\mathrm{XR}$ \\
\hline Mollugo verticillata $\mathrm{L}$. & Emergent & $\mathrm{XR}$ \\
\hline \multicolumn{3}{|l|}{ Myrtaceae } \\
\hline Myrcia guianensis (Aubl.) DC. & Amphibious & LA \\
\hline Myrcia splendens (SW.) DC. & Amphibious & $\mathrm{AF}$ \\
\hline Psidium acutangulum DC. & Amphibious & $\mathrm{XR}$ \\
\hline \multicolumn{3}{|l|}{ Nymphaeaceae } \\
\hline Nymphaea amazonum Mart. \& Zucc. ssp amazonum & Rooted floating & $\mathrm{AF}$ \\
\hline Nymphaea gardneriana Planch. & Rooted floating & $\mathrm{LA}, \mathrm{AF}$ \\
\hline \multicolumn{3}{|l|}{ Onagraceae } \\
\hline Ludwigia affinis (DC.) H. Hara & Amphibious & $\mathrm{LA}, \mathrm{AF}$ \\
\hline Ludwigia hyssopifolia (G.Don) Excell & Amphibious & LA, XR, AF \\
\hline Ludwigia leptocarpa (Nutt.) Hara & Emergent & $\mathrm{LA}, \mathrm{XR}, \mathrm{AF}$ \\
\hline Ludwigia nervosa (Poir.) & Emergent or Amphibious & $\mathrm{AF}$ \\
\hline Ludwigia octovalvis (Jacq.) P.H. Raven & Amphibious & $\mathrm{LA}, \mathrm{AF}$ \\
\hline \multicolumn{3}{|l|}{ Oxalidaceae } \\
\hline Oxalis barrelieri $\mathrm{L}$. & Amphibious & $\mathrm{XR}$ \\
\hline \multicolumn{3}{|l|}{ Parkeriaceae } \\
\hline Ceratopteris pteridoides (Hook.) & Free floating & $\mathrm{LA}, \mathrm{AF}$ \\
\hline \multicolumn{3}{|l|}{ Phyllanthaceae } \\
\hline Phyllanthus niruri L. & Amphibious & XR \\
\hline \multicolumn{3}{|l|}{ Piperaceae } \\
\hline Peperomia pellucida L. & Emergent & LA, XR \\
\hline \multicolumn{3}{|l|}{ Plantaginaceae } \\
\hline Bacopa rotundifolia (Michx.) Wettst. & Rooted floating & $\mathrm{AF}$ \\
\hline Scoparia dulcis L. & Amphibious & $\mathrm{LA}, \mathrm{AF}$ \\
\hline \multicolumn{3}{|l|}{ Poaceae } \\
\hline Acroceras zizanioides (H.B.K.) Dandy & Emergent or Amphibious & LA \\
\hline Andropogon bicornis $L$. & Emergent & $\mathrm{LA}, \mathrm{AF}$ \\
\hline
\end{tabular}

LA: lagoons; XR: Xingu River; AF: affluents. 
Table 1. Continued...

\begin{tabular}{|c|c|c|}
\hline Family/Species & Life form & Habitat \\
\hline Digitaria ciliaris (Retz.) Koeler & Amphibious & $\mathrm{XR}$ \\
\hline Echinochloa colona (L.) Link & Emergent & $\mathrm{XR}, \mathrm{AF}$ \\
\hline Echinochloa crusgalii (L.) P. Beauv. & Emergent & $\mathrm{XR}, \mathrm{AF}$ \\
\hline Echinochloa polystachya (H.B.K.) Hitchc. & Amphibious & $\mathrm{XR}$ \\
\hline Eragrostis japonica (Thunb.) Trin. & Amphibious & $\mathrm{XR}, \mathrm{AF}$ \\
\hline Homolepsis aturensis (Kunth) Chase & Amphibious & $\mathrm{AF}$ \\
\hline Hymenachne amplexicaulis (Rudge) Nees & Emergent & $\mathrm{LA}, \mathrm{XR}, \mathrm{AF}$ \\
\hline Luziola subintegra Swallen & Emergent or Amphibious & $\mathrm{XR}$ \\
\hline Megathyrsus massimus (Jacq.) BK Simon \& SWL Jacobs & Emergent & $\mathrm{XR}, \mathrm{AF}$ \\
\hline Olyra ecaudata Döll. & Amphibious & LA \\
\hline Oryza glumaepatula Steud. & Emergent & $\mathrm{XR}$ \\
\hline Panicum aquaticum Poir. & Emergent & $\mathrm{AF}$ \\
\hline Panicum dichotomiflorum Michx. & Emergent & $\mathrm{AF}$ \\
\hline Panicum elephantipes Nees ex Trin. & Emergent & $\mathrm{AF}$ \\
\hline Panicum repens L. & Emergent & LA \\
\hline Paspalum conjugatum P.J. Bergius & Emergent & $\mathrm{AF}$ \\
\hline Paspalum conspersum Schrad. & Emergent & $\mathrm{LA}, \mathrm{XR}, \mathrm{AF}$ \\
\hline Paspalum repens P. J. Bergius & Emergent & $\mathrm{LA}, \mathrm{XR}, \mathrm{AF}$ \\
\hline Paspalum virgatum $\mathrm{L}$. & Emergent & LA, XR \\
\hline Stephostachys mertensii (Roth) Zuloaga \& Morrone & Emergent & $\mathrm{LA}, \mathrm{AF}$ \\
\hline \multicolumn{3}{|l|}{ Podostemaceae } \\
\hline Castelnavia princeps Tul. \&Wedd. & Emergent/rooted submerged & $\mathrm{XR}$ \\
\hline Mourera alcicornis (Tul.) P.Royen & Emergent/rooted submerged & $\mathrm{XR}$ \\
\hline Mourera elegans (Tul.) Baill. & Emergent/rooted submerged & XR \\
\hline Mourera fluviatilis Aubl. & Emergent/rooted submerged & $\mathrm{XR}$ \\
\hline Tristicha trifaria (Bory ex Willd.) Spreng. & Emergent/rooted submerged & $\mathrm{XR}$ \\
\hline Weddellina squamulosaTul. & Emergent/rooted submerged & $\mathrm{XR}$ \\
\hline \multicolumn{3}{|l|}{ Polygalaceae } \\
\hline Polygala paniculata $\mathrm{L}$. & Emergent or Amphibious & $\mathrm{XR}$ \\
\hline \multicolumn{3}{|l|}{ Polygonaceae } \\
\hline Polygonum acuminatum Kunth & Amphibious & LA \\
\hline Polygonum punctatum Elliott & Emergent or Amphibious & $\mathrm{LA}, \mathrm{AF}$ \\
\hline Polygonum sp. & Emergent & $\mathrm{LA}, \mathrm{XR}, \mathrm{AF}$ \\
\hline Symmeria paniculata Benth. & Amphibious & $\mathrm{AF}$ \\
\hline \multicolumn{3}{|l|}{ Pontederiaceae } \\
\hline Eichhornia azurea (Sw.) Kunth & Rooted floating & $\mathrm{LA}, \mathrm{AF}$ \\
\hline Eichhornia crassipes (Mart.) Solms & Free floating & LA, XR \\
\hline Eichhornia diversiflora (Vahl) Urb. & Rooted floating & $\mathrm{AF}$ \\
\hline Pontederia subovata (Seub.) Lowden & Rooted floating & LA \\
\hline \multicolumn{3}{|l|}{ Rubiaceae } \\
\hline Alibertia latifolia (Benth.) K. Schum. & Amphibious & LA \\
\hline Borreria capitata Ruiz \& Pav. & Emergent & $\mathrm{XR}$ \\
\hline Borreria latifolia (Aubl.) K.Schum. & Amphibious & $\mathrm{AF}$ \\
\hline Borreria ocymoides (Burm.f) DC. & Amphibious & $\mathrm{LA}, \mathrm{AF}$ \\
\hline Borreria venticillata $\mathrm{L}$. & Amphibious & $\mathrm{LA}, \mathrm{XR}, \mathrm{AF}$ \\
\hline Diodia kuntzei K. Schum. & Amphibious & LA, XR \\
\hline Mitracarpus hirtus (L.) DC. & Emergent & XR \\
\hline Oldenlandia tenuis (K. Schum.) DC. & Amphibious & $\mathrm{AF}$ \\
\hline Sipanea pratensis Aubl. & Amphibious & $\mathrm{LA}, \mathrm{XR}, \mathrm{AF}$ \\
\hline Staelia reflexa DC. & Amphibious & XR \\
\hline
\end{tabular}

LA: lagoons; XR: Xingu River; AF: affluents. 
Table 1. Continued...

\begin{tabular}{|c|c|c|}
\hline Family/Species & Life form & Habitat \\
\hline \multicolumn{3}{|l|}{ Rutaceae } \\
\hline Esenbeckia grandiflora Mart. & Amphibious & XR \\
\hline \multicolumn{3}{|l|}{ Sapindaceae } \\
\hline Cupania latifolia Kunth. & Amphibious & $\mathrm{AF}$ \\
\hline Paullinia pinnata $\mathrm{L}$. & Amphibious & $\mathrm{XR}, \mathrm{AF}$ \\
\hline \multicolumn{3}{|l|}{ Solanaceae } \\
\hline Physalis angulata L. & Amphibious & $\mathrm{XR}$ \\
\hline Solanum crinitum Lam. & Amphibious & $\mathrm{AF}$ \\
\hline Solanum jamaicense Mill. & Amphibious & $\mathrm{XR}, \mathrm{AF}$ \\
\hline Solanum viarum Dunal & Amphibious & $\mathrm{AF}$ \\
\hline \multicolumn{3}{|l|}{ Turneraceae } \\
\hline Piriqueta cistoides (L.) Griseb. & Amphibious & LA, XR \\
\hline Piriqueta cistoides subsp. caroliniana (Walt.) Arbo & Amphibious & $\mathrm{XR}$ \\
\hline \multicolumn{3}{|l|}{ Verbanaceae } \\
\hline Lantana canescens Kunth & Amphibious & LA \\
\hline Stachytharpeta angustifolia (Mill.) Vahl. & Amphibious & $\mathrm{LA}, \mathrm{AF}$ \\
\hline \multicolumn{3}{|l|}{ Vitaceae } \\
\hline Cissus erosa Rich. & Amphibious & $\mathrm{LA}, \mathrm{XR}, \mathrm{AF}$ \\
\hline
\end{tabular}

LA: lagoons; XR: Xingu River; AF: affluents.

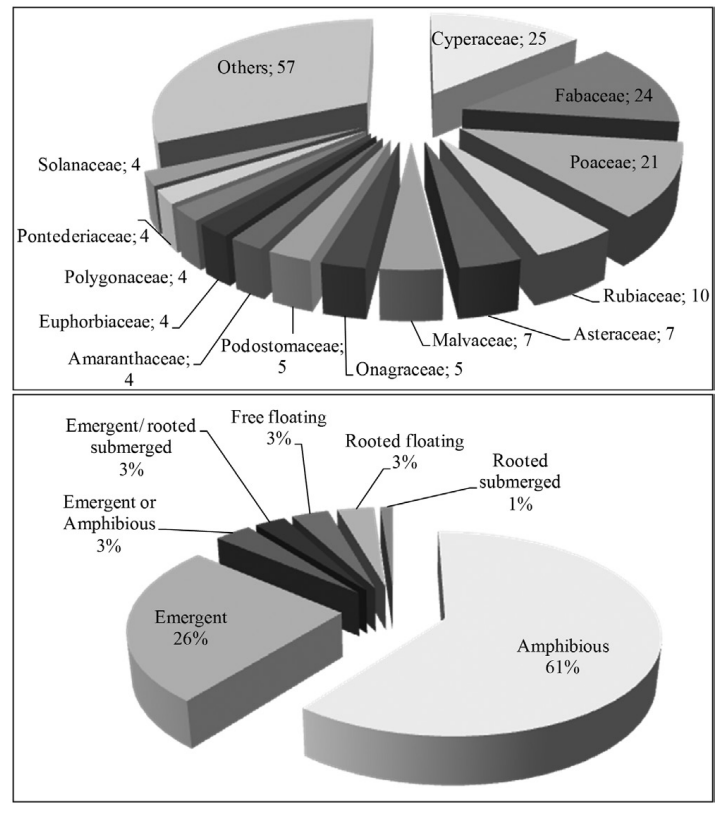

Figure 2. Life forms and proportion of families of aquatic macrophytes in the riverine landscapes of the Xingu River.

\section{Results}

Table 1 shows the additional list of species of macrophytes found in the lower basin of the Xingu River.

\section{Discussion}

Although the number of species observed in the present study was higher than that observed by Medeiros et al. (2015), who registered 106 species over two years of monitoring, the proportion of life forms was similar. The high number of amphibian species observed in this study is due to greater efforts directed to the characterization of plants living in the fllodplains and in the parafluvial zone of the Xingu River and tributaries. These results show the importance of these areas as habitats for aquatic macrophytes in the Xingu River basin.

\section{Acknowledgements}

The authors wish to express their thanks to Norte Energia S. A. for support and funding of the project, and Lisbeth Podanoschi Faria and Haren Santos for helping during the field campaigns.

\section{References}

Brasil. Ministério do Meio Ambiente - MMA, 2008. Instrução Normativa no 06, de 23 de setembro de 2008. Diário Oficial da República Federativa do Brasil, Brasília, 55 p.

BUCK, WR. and GOFFINET, B. 2000. Morphology and classification of mosses. In Shaw, JA. and Goffinet, B. (Eds.). Bryophyte Biology. Cambridge: Cambridge University Press. p. 72-124. http://dx.doi.org/10.1017/CBO9781139171304.004.

MEDEIROS, GR., RODRIGUES-FILHO, JL., MATSUMURATUNDISI, T., TUNDISI, JEM., ABE, DS, OLIVEIRA, HA., DEGANI, RM., BLANCO, FP., FARIA, CRL., CAMPANELLI, L., SOARES, FS., SIDAGIS-GALLI, C., TEIXEIRA-SILVA, V., GATTI-JUNIOR, P. and TUNDISI, JG. 2015. Occurrence of Macrophytes species in the lower basin of the Xingu River. Brazilian Journal of Biology. In press.

MOURA JÚNIOR, EG., PAIVA, RMS., FERREIRA, AC., PACOPAHYBA, LD., TAVARES, AS., FERREIRA, FA. and POTT, A., 2015. Updated checklist of aquatic macrophytes from 
Northern Brazil. Acta Amazonica, vol. 45, no. 2, p. 111-132. http:// dx.doi.org/10.1590/1809-4392201402662.

Reflora, 2015. Lista de espécies da flora do Brasil. Rio de Janeiro: Jardim Botânico do Rio de Janeiro. Available from: $<\mathrm{http}: / /$ floradobrasil.jbrj.gov.br/>. Access in: 20 July 2015.

SMITH, AR., PRYER, KM., SCHUETTPELZ, E., KORALL, P., SCHNEIDER, H. and WOLF, PG., 2006. A classification for extant ferns. Taxon, vol. 55, no. 3, p. 705-731. http://dx.doi. org $/ 10.2307 / 25065646$.

The Angiosperm Phylogeny Group - APG III, 2009. An update of the Angiosperm Phylkogeny Group classification for the orders and families of flowering plants: APG III. Botanical Journal of the Linnean Society, vol. 161, no. 2, p. 105-121. http://dx.doi. org/10.1111/j.1095-8339.2009.00996.x. 